\title{
Modeling pentaquark and heptaquark states
}

\author{
M. Nuñez V., S. Lerma H., and P. O. Hess \\ Instituto de Ciencias Nucleares, Universidad Nacional Autónoma de México, Apdo. Postal 70-543, México 04510 D.F. \\ S. Jesgarz \\ Instituto de Fisica, Unversidade de São Paulo, CP 66318, São Paulo 05315-970, SP, Brasil \\ O. Civitarese and M. Reboiro \\ Departamento de Física, Universidad Nacional de La Plata, c.c. 67 1900, La Plata, Argentina
}

(Received 29 January 2004; published 23 August 2004)

\begin{abstract}
A schematic model for hadronic states, based on constituent quarks and antiquarks and gluon pairs, is discussed. The phenomenological interaction between quarks and gluons is QCD motivated. The obtained hadronic spectrum leads to the identification of nucleon and $\Delta$ resonances and to pentaquark and heptaquark states. The predicted lowest pentaquark state $\left(J^{\pi}=\frac{1}{2}^{-}\right)$lies at the energy of $1.5 \mathrm{GeV}$ and it is associated to the observed $\Theta^{+}(1540)$ state. For heptaquarks $\left(J^{\pi}=\frac{1}{2}{ }^{+}, \frac{3}{2}{ }^{+}\right)$the model predicts the lowest state at $2.5 \mathrm{GeV}$.
\end{abstract}

DOI: 10.1103/PhysRevC.70.025201

PACS number(s): $24.85 .+\mathrm{p}, 12.39 .-\mathrm{x}, 14.20 .-\mathrm{c}$

\section{INTRODUCTION}

In a series of previous publications [1-3] a schematic model for QCD was developed. The model was used to test the meson spectrum of QCD. In spite of its schematic nature the model seems to contain the relevant degrees of freedom (color, flavor, and spin), as it was shown in the comparison between calculated and experimental meson spectra [2]. This letter is devoted to the extension of the model to accommodate baryonic features. Particularly, we shall concentrate on the appearance of exotic baryonic states, like pentaquark and heptaquark states [4-8].

The essentials of the model were discussed in detail in Ref. [2]. It consists of two fermionic levels in the quark $(q)$ and antiquark $(\bar{q})$ sector and a gluonic $\left(g^{2}\right)$ state containing pairs of gluons. These are the elementary building blocks of the model. The interaction among these parts is described by excitations of pairs of quarks and antiquarks mediated by the exchange of pairs of gluons. The pairs of quarks are classified in a flavor-spin coupling scheme. The pairs of gluons are kept in the angular momentum $(J)$, parity $(\pi)$, and charge conjugation $(C)$ state $J^{\pi C}=0^{++}$. The strength of various channels of the interaction, as well as the constituent masses, are taken from a phenomenological analysis. The model describes meson $\left((q \bar{q})^{n}\left(g^{2}\right)^{m}\right)$ states and baryonic $\left(q^{3}(q \bar{q})^{n}\left(g^{2}\right)^{m}\right)$ states. Among these states we focus on $q^{3}(q \bar{q})$ states (pentaquarks) and $q^{3}(q \bar{q})^{2}$ states (heptaquarks), where the configurations indicated represent the leading terms in an expansion over many quark-antiquark and gluon states. The basis states are classified using group theoretical methods [2]. The interaction of quark-antiquark pairs with gluon pairs is particle nonconserving.

The above described model belongs to a class of exactly solvable models of coupled fermion and boson systems [9-12]. Alternative descriptions of pentaquark states, which use also an algebraic approach but which include orbital excitations, and enforce particle number conservation, have been proposed in Ref. [13].
In what follows we shall classify the basis states and solve the Hamiltonian in the framework of the boson expansion method $[14,15]$. Finally, we shall compare the results of the calculations with recently published experimental data [4-7].

The model Hamiltonian is written

$$
\begin{aligned}
\boldsymbol{H}= & 2 \boldsymbol{\omega}_{f} \boldsymbol{n}_{\boldsymbol{f}}+\omega_{b} \boldsymbol{n}_{\boldsymbol{b}}+\sum_{\lambda S} V_{\lambda S}\left\{\left[\left(\boldsymbol{b}_{\lambda S}^{\dagger}\right)^{2}+2 \boldsymbol{b}_{\lambda S}^{\dagger} \boldsymbol{b}_{\lambda S}+\left(\boldsymbol{b}_{\lambda S}\right)^{2}\right]\right. \\
& \left.\times\left(1-\frac{\boldsymbol{n}_{\boldsymbol{f}}}{2 \Omega}\right) \boldsymbol{b}+\boldsymbol{b}^{\dagger}\left(1-\frac{\boldsymbol{n}_{f}}{2 \Omega}\right)\left[\left(\boldsymbol{b}_{\lambda S}^{\dagger}\right)^{2}+2 \boldsymbol{b}_{\lambda S}^{\dagger} \boldsymbol{b}_{\lambda S}+\left(\boldsymbol{b}_{\lambda S}\right)^{2}\right]\right\} \\
& +\boldsymbol{n}_{(0,1) 0}\left(D_{1} \boldsymbol{n}_{\boldsymbol{b}}+D_{2}\left(\boldsymbol{b}^{\dagger}+\boldsymbol{b}\right)\right)+\boldsymbol{n}_{(2,0) 1}\left(E_{1} \boldsymbol{n}_{\boldsymbol{b}}+E_{2}\left(\boldsymbol{b}^{\dagger}+\boldsymbol{b}\right)\right) .
\end{aligned}
$$

The distance between the fermion levels is $2 \omega_{f}=0.66 \mathrm{GeV}$, $\omega_{b}=1.6 \mathrm{GeV}$ is the energy of the glue ball, $\boldsymbol{n}_{\boldsymbol{f}}$ and $\boldsymbol{n}_{\boldsymbol{b}}$ are the number operators for fermion and gluon pairs, respectively, $V_{\lambda S}$ is the strength of the interaction in the flavor $(\lambda)$ and spin $(S)$ channel. The actual values $\lambda=0,1$ refer to flavor $(0,0)$ and $(1,1)$ configurations, while the spin channel is $S$ $=0$ or 1 . The adopted values are: $V_{00}=0.0337 \mathrm{GeV}, V_{01}$ $=0.0422 \mathrm{GeV}, V_{10}=0.1573 \mathrm{GeV}$, and $V_{11}=0.0177 \mathrm{GeV}$ [2]. The operators $\boldsymbol{b}_{\lambda S}^{\dagger}$ and $\boldsymbol{b}_{\lambda S}$ are boson images of quarkantiquark pairs [2]. The products which appear inside brackets in (1) are scalar products. The factor $\left(1-\boldsymbol{n}_{f} / 2 \Omega\right)$ results from the boson mapping [2]. The mapping is exact for the channel $[\lambda, S]=[0,0]$ and simulates the effect of the boson mapping for the other channels. The operator $\boldsymbol{b}^{\dagger}(\boldsymbol{b})$ creates (annihilates) gluon pairs with spin-color zero, and $\boldsymbol{n}_{\left(\lambda_{0}, \mu_{0}\right) S_{0}}$ is the number operator of di-quarks coupled to flavor-spin $\left(\lambda_{0}, \mu_{0}\right) S_{0}$. The parameters $D_{1(2)}$ and $E_{1(2)}$ are adjusted to reproduce the nucleon and $\Delta$ resonances. The corresponding terms describe the interaction between valence quarks and mesons. The Hamiltonian (1) does not contain terms which distinguish between states with different hypercharge and isospin. It does not contain flavor mixing terms, either. Therefore, the predicted states have to be corrected in 
the way described in Ref. [16] to allow a comparison with data. The adopted values of $D_{1(2)}$ and $E_{1(2)}$ are: $D_{1}$ $=-1.442 \mathrm{GeV}, D_{2}=-0.4388 \mathrm{GeV}, E_{1}=-1.1873 \mathrm{GeV}$, and $E_{2}=-0.3622 \mathrm{GeV}$. The Hamiltonian contains all relevant degrees of freedom requested by QCD.

The complete classification of quark-antiquark configurations was given in Ref. [2].

The unperturbed ground state is composed by 18 quarks occupying the lowest fermionic level. The baryonic states are described by three quarks in the upper fermionic level to which we add $(q \bar{q})^{n}$ states. The group chain which describes these states is

$$
\begin{gathered}
{\left[1^{N}\right][h]=\left[h_{1} h_{2} h_{3}\right]\left[h^{T}\right],} \\
U(4 \Omega) \supset U\left(\frac{\Omega}{3}\right) \otimes U(12),
\end{gathered}
$$

where $\Omega=9$ accounts for three color and three flavor degrees of freedom. The irreducible representation (irrep) of $\mathrm{U}(4 \Omega)$ is completely antisymmetric, and $\left[h^{T}\right]$ is the transposed Young diagram of [h] [17]. For $N$ particles, and due to the antisymmetric irrep $\left[1^{N}\right]$ of $\mathrm{U}(4 \Omega)$, the irreps of $\mathrm{U}(\Omega / 3)$ and $\mathrm{U}(12)$ are complementary and the irrep of $\mathrm{U}(\Omega / 3)$ is the color group, which is reduced to $\mathrm{SU}_{C}(3)$ with the color irrep $\left(\lambda_{C}, \mu_{C}\right)$. The $\mathrm{U}(12)$ group is further reduced to

$$
\begin{gathered}
\mathrm{U}(12) \supset \mathrm{U}_{f}(3) \otimes \mathrm{U}(4) \supset \mathrm{SU}_{f}(3) \otimes \mathrm{SU}_{S}(2) \\
{\left[p_{1} p_{2} p_{3} p_{4}\right]\left(\lambda_{f}, \mu_{f}\right) \quad S, M,}
\end{gathered}
$$

where $\left(\lambda_{f}, \mu_{f}\right)$ is the flavor irrep and $\left[p_{1} p_{2} p_{3} p_{4}\right]$ denotes the possible $\mathrm{U}(4)$ irreps. The group reduction is done using the methods exposed in Ref. [18]. The basis is spanned by the states

$$
\left|N,\left[p_{1} p_{2} p_{3} p_{4}\right]\left(\lambda_{C}, \mu_{C}\right), \rho_{f}\left(\lambda_{f}, \mu_{f}\right) Y T T_{z}, \rho_{S} S M\right\rangle,
$$

where $N$ is the number of particles, $Y$ is the hypercharge, and $\left(T, T_{z}\right)$ denotes the isospin and its third component, $\rho_{f}$ and $\rho_{S}$ are the multiplicities of the flavor and spin representations. The color labels $\left(\lambda_{C}, \mu_{C}\right)$ are related to the $h_{i}$ via $\lambda_{C}=h_{1}$ $-h_{2}$ and $\mu_{C}=h_{2}-h_{3}$. To obtain the values of $h_{i}$ one has to consider all possible partitions of $N=h_{1}+h_{2}+h_{3}$, which fixes the color. For colorless states we have $h_{1}=h_{2}=h_{3}=h$. Each partition of $N$ appears only once. The irrep [hhh] of $\mathrm{U}(\Omega / 3)=\mathrm{U}(3)$ fixes the irrep of $\mathrm{U}(12)$, as indicated in (2). For $\Omega=9$ and color $(0,0)$ the irrep of $\mathrm{U}(12)$ is given by $\left[3^{6} 0^{6}\right]$ for mesons, and by $\left[3^{7} 0^{5}\right]$ for baryons. As an example, Table I shows the relevant irreps for mesonic states. (More details are given in Ref. [19].)

In the boson representation, the states are given by the direct product of one-, three-, eight- and 24-dimensional harmonic oscillators [2]. For each harmonic oscillator the basis states are given by

$$
\mathcal{N}_{N_{\lambda S} \nu_{\lambda S}}\left(\boldsymbol{b}_{\lambda S}^{\dagger 2}\right)^{N_{\lambda S}-\nu_{\lambda S} / 2}\left|\nu_{\lambda S} \alpha_{\lambda S}\right\rangle
$$

where $N_{\lambda S}$ is the number of bosons of type $[\lambda, S], \nu_{\lambda S}$ is the corresponding seniority, and $\mathcal{N}_{N_{\lambda S} \nu_{\lambda S}}$ is a normalization constant. The seniority is defined as the number of uncoupled
TABLE I. Flavor irreps coupled to the quark-antiquark content of some different U(4) irreps. Shown are the irreps which contain, at most, two quarks and two antiquarks. The number of quarks (antiquarks) in a given configuration are denoted by $n_{q}\left(n_{q}\right)$.

\begin{tabular}{lcccccccc}
\hline \hline $\mathrm{SU}_{f}(3)$ & $\mathrm{U}(4)$ & {$\left[q_{1} q_{2}\right]$} & $n_{q}$ & $S_{q}$ & {$\left[\overline{q_{1}} \overline{q_{2}}\right]$} & $n_{q}^{-}$ & $S_{\bar{q}}$ & $S$ \\
\hline$(0,0),(1,1),(2,2)$ & {$[8811]$} & {$[11]$} & 2 & 0 & {$[88]$} & 2 & 0 & 0 \\
$(1,1),(3,0),(0,3)$ & {$[9711]$} & {$[11]$} & 2 & 0 & {$[97]$} & 2 & 1 & 1 \\
$(1,1),(3,0),(0,3)$ & {$[8820]$} & {$[20]$} & 2 & 1 & {$[88]$} & 2 & 0 & 1 \\
$(0,0),(1,1),(2,2)$ & {$[9720]$} & {$[20]$} & 2 & 1 & {$[97]$} & 2 & 1 & $0,1,2$ \\
$(1,1)$ & {$[9810]$} & {$[10]$} & 1 & $\frac{1}{2}$ & {$[98]$} & 1 & $\frac{1}{2}$ & 0,1 \\
$(1,1)$ & {$[9810]$} & {$[11]$} & 2 & 0 & {$[97]$} & 2 & 1 & 1 \\
$(1,1)$ & {$[9810]$} & {$[11]$} & 2 & 0 & {$[88]$} & 2 & 0 & 0 \\
$(1,1)$ & {$[9810]$} & {$[20]$} & 2 & 1 & {$[97]$} & 2 & 1 & $0,1,2$ \\
$(1,1)$ & {$[9810]$} & {$[20]$} & 2 & 1 & {$[88]$} & 2 & 0 & 1 \\
$(0,0)$ & {$[9900]$} & {$[00]$} & 0 & 0 & {$[99]$} & 0 & 0 & 0 \\
$(0,0)$ & {$[9900]$} & {$[10]$} & 1 & $\frac{1}{2}$ & {$[98]$} & 1 & $\frac{1}{2}$ & 0,1 \\
$(0,0)$ & {$[9900]$} & {$[20]$} & 2 & 1 & {$[97]$} & 2 & 1 & $0,1,2$ \\
\hline \hline
\end{tabular}

bosons. The quantity $\alpha_{\lambda S}$ represents the other quantum numbers needed to specify a particular harmonic oscillator.

Concerning the width of the states, in the present version of the model it cannot be calculated. For it, one has to construct the explicit form of $\left|\nu_{\lambda S} \alpha_{\lambda S}\right\rangle$ and calculate the overlap of the initial state with the states of the exit channel, which can be seen as a cluster component of the initial state. In spite of this limitation we can advance some qualitative arguments. We can determine the average numbers of quarkantiquark pairs coupled to a definite flavor and spin and also the average number of gluon pairs coupled to color and spin zero. If these average numbers, as obtained for the initial state, differ with respect to the sum of the average numbers of the particles in the decay channel, then the width of the state may be small. If these average numbers are similar, the appearance of a broad width may be expected.

\section{NUCLEON RESONANCES}

The quality of the model predictions, concerning meson states, was discussed in Ref. [2]. Figure 1 shows the lowest nucleon and $\Delta$ resonances predicted by the model. In the same figure are shown the calculated penta- and heptaquark low lying states. For each state we indicate the spin, parity $\left(J^{\pi}\right)$, and the quark and gluon content $\left(n_{q}+n_{\bar{q}}, n_{g}\right)$. The quantity $n_{q}+n_{\bar{q}}$ is the total number of quarks and antiquarks, which is equal to the number of valence quarks ( 0 for mesons, 3 baryons) plus the number of quarks and antiquarks of the $q \bar{q}$ pairs, and $n_{g}$ gives the number of gluons. As shown in the figure, nucleonic states contain on the average about half an additional quark-antiquark pair (equivalent to one extra quark), and approximately 2.8 gluons. This implies a content of $59 \%$ in the quark sector and of $41 \%$ in the gluon sector. The theoretical Roper resonance (first excited nucleon resonance) lies near the experimental energy of $1.44 \mathrm{GeV}$. Most constituent models predict the Roper resonance too high, above the negative-parity excitation. An exception is the 


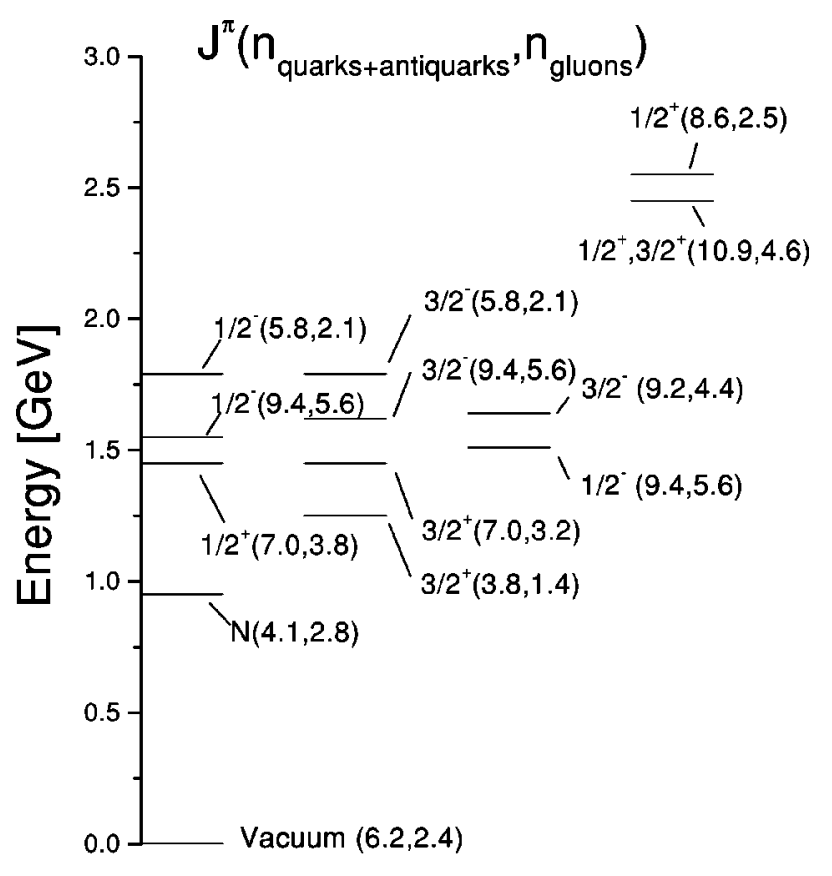

Calculated Spectrum

FIG. 1. Nucleon resonances (first group of levels), $\Delta$ resonances (second group), pentaquarks (third group) and heptaquarks (fourth group). On the right side of each level are given the assigned spin and parity $\left(J^{\pi}\right)$, and the total quark and antiquark $\left(n_{q}+n_{q}\right)$ and gluon $\left(n_{g}\right)$ contents (see the text).

model of [20], where the energy of the Roper resonance is adjusted in a particular dynamical symmetry limit. In our approach the Roper resonance appears at low energy because of the sudden increase of the particle content of the configuration, a feature which results in a collective excitation. The first negative parity state with $J^{\pi}=\frac{1}{2}{ }^{-}$appears at $1.51 \mathrm{GeV}$, also in good agreement with the data.

\section{III. $\Delta$ RESONANCES}

The simplest way to obtain a $\Delta$ resonance is to couple the three valence quarks in the $(3,0) \frac{3}{2}$ configuration with $q \bar{q}$ pairs in a $(0,0) J=0$ configuration. This scheme leads to the $\Delta(1232)$ resonance. The quark-antiquark and gluon content of the calculated $\Delta(1232)$ turns out to be lower than that of the nucleon, while the structure of the state at $1.57 \mathrm{GeV}$ can be compared with the Roper resonance. Concerning negative parity states, Fig. 1 shows a $\frac{3}{2}^{-}$state at $1.79 \mathrm{GeV} . \Delta$ resonances can also be obtained by coupling the three valence quarks in the $(1,1) \frac{1}{2}$ configuration with $(1,1) S q \bar{q}$ states. The lowest state of this type is at $1.51 \mathrm{GeV}$, and it should be compared with the experimental value ( $\Delta$ resonance) at $1.62 \mathrm{GeV}[21]$.

\section{PENTAQUARKS AND HEPTAQUARKS}

In the present calculation the minimal representation of pentaquarklike states includes the following configurations:
$(0,0) \frac{1}{2}^{-},(1,1) \frac{1}{2}^{-},(3,0) \frac{1}{2}^{-},(0,3) \frac{1}{2}^{-}$and $(2,2) \frac{1}{2}^{-}$. Only the $(0,3) \frac{1}{2}-$ and $(2,2) \frac{1}{2}^{-}$configurations contain hypercharge and isospin combinations which cannot be obtained in a pure $q^{3}$ coupling scheme, like $T=0, Y=2$ in $(0,3)$ and $T=1, Y=2$ in $(2,2)$. Within the model, the lowest pentaquark state $\Theta^{+}(1540)$ [4-8] is interpreted as a coupling of the three valence quarks in $(1,1) \frac{1}{2}^{+}$with the $q \bar{q}$ background in $(1,1) 0^{-}$to the final irrep $(1,1) \frac{1}{2}^{-}$. Thus, within our model, the calculated pentaquark state at $1.51 \mathrm{GeV}$ may correspond to the observed $\Theta^{+}(1540)$ state [4-8]. Another predicted pentaquark state is shown in Fig. 1.

Within the model, the lowest pentaquark has negative parity in accordance with QCD sum-rules and lattice gauge calculations [22-25]. If the orbital spin $L$ is included, pentaquark states with positive parity may exist with $L=1$. However, these states include an orbital excitation and should appear at higher energies.

In order to obtain some information about the width of the lowest pentaquark state, we use the procedure mentioned before (see Sec. III). The decay channel considered is the emission of a Kaon and a nucleon. The distribution of quarkantiquark pairs in the lowest pentaquark state turns out to be just the sum of the distributions of a Kaon and a nucleon. This result suggests the existence of a very broad width, but it has to be taken with caution because internal couplings can change the situation.

The model contains heptaquarks, characterized by two $q \bar{q}$ pairs added to the three valence quarks. The lowest state has an energy of approximately $2.5 \mathrm{GeV}$ and it has a content of $3.9 q \bar{q}$ pairs of the type $(1,1) 1^{+}$coupled to the three valence quarks in the configuration $(1,1) \frac{1}{2}^{+}$. This coupling scheme yields three exotic flavor irreps: $(3,3) \frac{1}{2}{ }^{+}, \frac{3}{2}+,(4,1) \frac{1}{2}{ }^{+}, \frac{3}{2}+$, and $(1,4) \frac{1}{2}+\frac{3}{2}+$. The lowest heptaquark state contains, basically, three ideal valence quarks, four $q \bar{q}$ pairs and 4.6 gluons. This implies a quark content of $70 \%$ and a gluon content of $30 \%$. The model predicts other heptaquark states at higher energies, which are obtained by coupling the three ideal valence quarks with the $(3,0) 1^{+}$and $(0,3) 1^{+} q \bar{q}$ configurations. This leads to exotic flavor irreps like $(4,1),(1,4)$, and $(3,3)$ with spin $\frac{1}{2}^{+}$and $\frac{3}{2}^{+}$. The coupling of the three valence quarks with a $q \bar{q}$ irrep $(2,2) S=0,1,2$ leads to exotic flavor irreps of the type $(3,3),(1,4)$ with spin-parity $\frac{1}{2}^{+}, \frac{3}{2}^{+}$, and $\frac{5}{2}^{+}[19]$.

\section{HIGHER MULTIQUARK STATES}

In this letter we do not go further into the discussion of all possible states with the structure $q^{3}(q \bar{q})^{n_{2}} g^{n_{3}}$, since the number of these configurations increases with the energy. A more complete overview of these states will be presented in Ref. [19] with its complete classification of states.

To conclude, we have applied a schematic model based on QCD degrees of freedom, to describe nucleon and $\Delta$ resonances and more exotic penta- and heptaquark states. The basis states were classified by applying group theoretical methods. The Hamiltonian, used in the calculations, was tested to the mesonic spectrum, nucleon and $\Delta$ resonances. After fixing the parameters in this manner, we have investigated the appearance of penta- and heptaquark states. The 
results of the calculations show that the model predicts reasonably well the $\Theta^{+}(1540)$ resonance. The lowest pentaquark state is obtained at an energy of approximately $1.5 \mathrm{GeV}$ and it has $J^{\pi}=\frac{1}{2}^{-}$. The lowest heptaquark state has an energy of approximately $2.5 \mathrm{GeV}$ and $J^{\pi}$ either $\frac{1}{2}^{+}$or $\frac{3}{2}^{+}$. In addition, other penta- and heptaquark states are predicted to appear at higher energies.

The model allows for a complete classification of many quark-antiquark and gluon systems. As we have shown, the exotic configurations which appear in our classification scheme cannot be obtained in a simple constituent quark picture. The overall agreement with the experimental data supports the claim about the suitability of the procedure.

\section{ACKNOWLEDGMENTS}

This work obtained financial support from the DGAPA Project No. IN119002 and from CONACyt, CONICET. S.J. thanks the German Academic Interchange Service (DAAD) for financial support.
[1] S. Lerma, S. Jesgarz, P. O. Hess, O. Civitarese, and M. Reboiro, Phys. Rev. C 66, 045207 (2002).

[2] S. Lerma, S. Jesgarz, P. O. Hess, O. Civitarese, and M. Reboiro, Phys. Rev. C 67, 055209 (2003).

[3] S. Jesgarz, S. Lerma, P. O. Hess, O. Civitarese, and M. Reboiro, Phys. Rev. C 67, 055210 (2003).

[4] T. Nakano et al., Phys. Rev. Lett. 91, 012002 (2003).

[5] V. V. Barmin et al. (DIANA collaboration), hep-ex/0304040 (unpublished); Yad. Fiz. (unpublished).

[6] S. Stepanyan et al. (CLAS collaboration), hep-ex/0307018 (unpublished).

[7] J. Barth et al. (SAPHIR collaboration), hep-ex/307083 (unpublished); J. Barth et al. Nucl. Phys. B (unpublished).

[8] A. E. Asratyan, A. G. Dolgolenko, and M. A. Kubantsev, hepex/0309042 (unpublished); Yad. Fiz. (unpublished).

[9] H. J. Lipkin, N. Meschkov, and S. Glick, Nucl. Phys. A62, 118 (1965).

[10] D. Schütte and J. Da Providencia, Nucl. Phys. A282, 518 (1977).

[11] J. Dobes and S. Pittel, Phys. Rev. C 57, 688 (1998).

[12] O. Civitarese and M. Reboiro, Phys. Rev. C 58, 2787 (1998).

[13] R. Bijker, M. M. Giannini, and E. Santopinto, hep-ph/0310281 (unpublished); hep-ph/0312380 (unpublished).

[14] A. Klein and E. R. Marshalek, Rev. Mod. Phys. 63, 375 (1991).

[15] J. G. Hirsch, P. O. Hess, and O. Civitarese, Phys. Rev. C 60, 064303 (1999).

[16] F. Gürsey and L. A. Radicati, Phys. Rev. Lett. 13, 173 (1964).

[17] M. Hamermesh, Group Theory and its Application to Physical Problems (Dover, New York, 1989).

[18] R. López, P. O. Hess, P. Rochford, and J. P. Draayer, J. Phys. A 23, L229 (1990).

[19] M. Nuñez, S. Lerma, P. O. Hess, S. Jesgarz, O. Civitarese, and M. Reboiro, Phys. Rev. C (unpublished).

[20] R. Bijker, F. Iachello, and A. Leviatan, Ann. Phys. (N.Y.) 236, 69 (1994).

[21] K. Hagi et al., The Particle Data Group, Phys. Rev. D 66, 010001 (2002).

[22] Shin-Lin Zhu, hep-ph/0307345 (unpublished).

[23] J. Sugiyama, T. Doi, and M. Oka, hep-ph/0309271 (unpublished).

[24] F. Csikor, Z. Fodor, S. D. Katz and T. G. Kovács, hep-lat/ 0309090 (unpublished).

[25] S. Sasaki, hep-lat/0310014 (unpublished). 